\title{
A PERCEPÇÃO DOS DISCENTES QUANTO A REAL CONTRIBUIÇÃO DO LABORATÓRIO DE HABILIDADES DE ENFERMAGEM DURANTE SUA FORMAÇÃO ACADÊMICA
}

\author{
Milena Colonhese Camargo \\ Universidade do Oeste Paulista - UNOESTE, Curso de Enfermagem, Presidente Prudente, SP. e-mail: \\ milenacolonhese@uol.com.br
}

\begin{abstract}
RESUMO
O estudo buscou conhecer a percepção dos discentes da graduação quanto a real contribuição do laboratório de enfermagem na sua formação acadêmica. Pesquisa descritiva com abordagem quantitativa, em que foi aplicado um instrumento constituído de 10 perguntas de múltipla escolha para 190 alunos da graduação em enfermagem que já fizeram uso do laboratório. As técnicas mais e menos realizadas foram respectivamente: curativo (185 alunos) e oxigenoterapia (46 alunos); A técnica considerada mais difícil foi: sondagem vesical masculina; a demonstração da técnica pelo professor no laboratório de enfermagem antes da prática foi considerada válida para o aprendizado por 182 alunos e os professores auxiliaram durante o treinamento, 143 alunos. 0 laboratório de enfermagem utilizado na disciplina de Semiologia e Semiotécnica foi considerado importante para o aprendizado por 185 dos participantes; 137 apontaram como fator desestimulante para utilização do laboratório, a falta de tempo para agendamento de aulas; 176 concordam que todas as disciplinas teórico-prática do curso devem utilizar o laboratório e 160 responderam que a necessidade de adquirir maior segurança no procedimento foi um dos fatores que mais estimulou a utilizar o laboratório. Na percepção dos discentes o laboratório de enfermagem contribui para sua formação acadêmica.
\end{abstract}

Palavras-chave: estudantes de enfermagem, procedimentos, ensino.

\section{THE STUDENTS' PERCEPTIONS ABOUT A REAL CONTRIBUTION IN THE HABILITIES NURSING LABORATORY DURING THE COLLEGE EDUCATION}

\begin{abstract}
The present study analyzes the perception of students graduation about the real contribution of the nursing laboratory in their academic training. Descriptive research with quantitative approach, in which it was applied an instrument consisting of 10 multiple choice questions to 190 students of undergraduate nursing that have already used the laboratory. The techniques most and least performed were: curative (185 students) and oxygen therapy (46 students); The technique was considered more difficult: urinary catheterization male; the demonstration of the technique by the teacher in the nursing laboratory before practice was considered valid for learning by 182 students and teachers helped during training, 143 students. The nursing laboratory used in Semiology and Semiotecnican was considered important for learning by 185 participants; 137 indicated as a discouraging factor for use of the laboratory, the lack of time for scheduling classes; 176 agree that the course theoretical and practical disciplines should use the laboratory and 160 responded to the need to develop greater confidence in the procedure was one of the factors that encouraged the use laboratory. In the perception of nursing students the laboratory contributes to their academic training.
\end{abstract}

Keywords: nursing students, proceeding, teaching. 


\section{INTRODUÇÃO}

Laboratório de Habilidades de Enfermagem (LHE) é uma sala ou conjunto de salas equipadas com manequins, simuladores, materiais e equipamentos semelhantes aos das unidades hospitalares bastante frequentados pelos alunos durante as aulas de disciplinas teórico-prática sendo considerado um valioso recurso no processo de ensino-aprendizagem de habilidades psicomotoras, liderança, autonomia, gerenciamento e tomada de decisão, onde o aluno pode experimentar testar, repetir, errar e corrigir livre da ansiedade e responsabilidade causadas pela presença de um paciente ${ }^{1-3}$.

O objetivo do processo de ensinoaprendizagem no LHE é fazer com que o aluno desenvolva as habilidades necessárias para a realização das técnicas corretamente, adquirir segurança e diminuir o impacto psicológico. Antes de o aluno atuar na assistência direta ao paciente, o professor demonstra o procedimento, em seguida o aluno simula a técnica utilizando os manequins, com a finalidade de manipular os materiais e se familiarizar com a situação ${ }^{3-5}$.

Um dos benefícios do ensino no LHE é possibilitar uma assistência segura e de qualidade, no aspecto psicológico o treinamento prévio das habilidades reduz 0 medo do discente no primeiro contato. É melhor o aluno primeiramente ser avaliado em uma situação simulada para posteriormente ser avaliado em situação real. Porém é destacado do ponto de vista ético e importante que, o ensino no laboratório de enfermagem não pode substituir o ensino na prática clínica, pois alguns aspectos da aprendizagem só podem ser desenvolvidos no contato aluno/paciente ${ }^{6}$.

Gagliazzi e Friedlander ${ }^{7}$, relatam em seu estudo que o treinamento prévio no LHE é muito importante para o aluno desenvolver competência e segurança, reduzindo os riscos à integridade física do paciente quando atendido em situação real. O primeiro contato com o paciente é uma situação que causa muita ansiedade e insegurança, provocando no aluno sintomas físicos que prejudicam seu aprendizado e desempenho e muitas vezes ficam tão assustados que não percebe o paciente como um todo, conseguindo apenas ver à sua frente o procedimento a ser realizado.

Algumas respostas emocionais como choro, medo, insegurança e angústia, são percebidas quando o aluno executa alguns procedimentos como curativos e administração de medicamentos por via parenteral, mesmo em situações simuladas no $\mathrm{LHE}^{3-8}$.

É necessário reconhecer que o ensino no LHE necessita de interação adequada entre os alunos e o professor. $\mathrm{O}$ docente da 
disciplina deverá estar capacitado e preparado para acolher o aluno e ajudá-lo a superar suas dificuldades e medos, suavizar os impactos neste primeiro momento de estresse que muitas vezes coloca em risco sua decisão sobre a profissão, tornando uma prática docente humanizada, formadora e ética ${ }^{5}$.

Os docentes das disciplinas introdutórias à prática, devem ter uma maior sensibilidade para entender os alunos com suas dificuldades e sentimentos durante $o$ desenvolvimento dos primeiros procedimentos para encorajá-lo a tornar um sujeito ativo do processo ensinar/aprender e não bloqueá-lo, deixando de preocupar-se apenas com a repetição mecânica dos procedimentos. O clima de respeito e confiança entre alunos e professores é fundamental para o aprendizado, pois o aluno sente-se a vontade para expressar suas dúvidas sem medo de ser repreendido ${ }^{5}$.

De acordo com Teixeira e Felix ${ }^{8}$, não podemos tolerar a ocorrência de erros com o paciente, sendo imprescindível a segurança para a aprendizagem dos procedimentos, e, nas práticas simuladas no LHE, os alunos podem errar para aprimorar as técnicas.

Apesar de a literatura demonstrar importância da utilização do LHE na formação do enfermeiro surge 0 seguinte questionamento: qual percepção dos alunos quanto à sua real efetividade? Dessa forma, justifica-se a realização desse trabalho no intuito de identificar os fatores que possam levar os discentes a não adesão das práticas propostas no referido ambiente e qual a real contribuição do laboratório de enfermagem na disciplina de Semiologia, Semiotécnica e Procedimentos do Cuidar como recurso de ensino na sua formação acadêmica.

\section{MÉTODOS}

Trata-se de uma pesquisa descritiva, transversal com abordagem quantitativa. Os sujeitos desta pesquisa foram os alunos do curso de graduação de Enfermagem de uma universidade do interior do estado de São Paulo que estavam presentes na aula no dia da aplicação do questionário de pesquisa, devidamente matriculados no $4^{\circ}, 5^{\circ}, 6^{\circ}, 7^{\circ} \mathrm{e}$ $8^{\circ}$ termos e que já concluíram a disciplina de Semiologia, Semiotécnica e Procedimentos do Cuidar.

Os participantes da pesquisa não têm mais atividades com os professores da referida disciplina e as características sóciodemográficas estudadas foram a idade o sexo e se trabalham na área da enfermagem.

Os aspectos éticos da pesquisa foram conduzidos de acordo com a Resolução 466/12 do Ministério da Saúde, após aprovação do Comitê de Ética em Pesquisa (CEP), protocolo $\mathrm{n}^{\circ} 1108$.

A coleta de dados foi realizada na primeira quinzena de setembro de 2012 , em 
que foi utilizado um instrumento elaborado pelos pesquisadores contendo 10 questões de múltipla escolha divididos em três eixos principais sendo:

- Habilidades desenvolvidas e grau de dificuldade: procedimentos desenvolvidos no laboratório de enfermagem e procedimentos em que o aluno mais sentiu dificuldade na realização;

- Importância do docente durante as práticas: a demonstração do procedimento pelo professor antes da prática é valida, não válida ou indiferente para o aprendizado e se o professor auxiliou durante o treinamento dos procedimentos.

- $\quad$ Percepção do aluno quanto à real utilidade deste tipo de atividade: a opinião quanto à importância sobre a utilização do LHE pela disciplina de Semiologia e Semiotécnica; a frequência de utilização do LHE para aulas de estudo; a disponibilidade de horário do LHE para aulas de estudo; a opinião sobre a utilização do LHE por todas as disciplinas teórico-prática do curso e fatores estimulantes e desestimulantes para o uso do LHE.

Os questionários foram aplicados em sala de aula, no período final das atividades acadêmicas após autorização prévia do professor logo depois os pesquisadores explicarem o objetivo da pesquisa. Os interessados em participar permaneceram na sala e após a assinatura do termo de Consentimento Livre e Esclarecido receberam o instrumento de coleta de dados e as orientações de preenchimento do mesmo. Depois de preencherem o instrumento de pesquisa, completamente sem identificação, os participantes colocaram o questionário em uma caixa lacrada (urna) que foi oferecida pelos pesquisadores.

As respostas dos estudantes foram agrupadas de acordo com os eixos e os dados foram tabulados através da construção de um banco de dados em planilhas do aplicativo Microsoft $\quad$ Excel $^{\circledR} \quad$ e subsequentemente foram analisados utilizando-se frequências simples e absolutas.

\section{RESULTADOS}

Participaram da pesquisa 190 alunos do curso de graduação de enfermagem, sendo que 85 (44,73\%) alunos não responderam a idade, $84(44,21 \%)$ encontrase na faixa etária dos 19 aos 30 anos (41,53\%), 168 (88,41\%) é do sexo feminino e $64(33,67 \%)$ já trabalham na área da enfermagem (Tabela 1).

Gagliazzi e Fridlander ${ }^{7}$ descreveram em sua pesquisa que a maior parte dos estudantes entrevistados encontrava-se na faixa etária dos 20 anos. Esses dados também foram descritos em um estudo envolvendo o processo de ensino-aprendizagem com alunos de graduação em enfermagem de Feliz et al. ${ }^{3}$, em que mais de $80 \%$ da amostra foi representado pelo sexo feminino e a idade predominante dos estudantes esteve entre 19 e 24 anos. 
Tabela 1. Distribuição dos participantes da pesquisa, segundo as características sociodemográficas.

\begin{tabular}{lcccccc}
\hline Características & $\begin{array}{c}\text { Trabalha na } \\
\text { área da } \\
\text { enfermagem }\end{array}$ & $\begin{array}{c}\text { Não trabalha } \\
\text { na área da } \\
\text { enfermagem }\end{array}$ & Total \\
\cline { 2 - 7 } & $\mathrm{f}$ & $\%$ & $\mathrm{f}$ & $\%$ & $\mathrm{f}$ & $\%$ \\
\hline Idade (anos) & 27 & $41,53 \%$ & 57 & $45,6 \%$ & 84 & $44,21 \%$ \\
$19-30$ & 8 & $12,30 \%$ & 9 & $7,2 \%$ & 17 & 8,94 \\
$31-40$ & 2 & $3,07 \%$ & 2 & $1,6 \%$ & 4 & $2,10 \%$ \\
$41-50$ & 28 & $43,07 \%$ & 57 & $45,6 \%$ & 85 & $44,73 \%$ \\
Não respondeu & 65 & $100 \%$ & 125 & $100 \%$ & 190 & $100 \%$ \\
\hline Total & 12 & 6,31 & 10 & 5,26 & 22 & 11,57 \\
\hline Sexo & 52 & 27,36 & 116 & 61,05 & 168 & 88,41 \\
Masculino & 64 & 33,67 & 126 & 66,31 & 190 & 100,0 \\
\hline Feminino & & & & & & \\
\hline Total & &
\end{tabular}

Com relação ao eixo habilidades desenvolvidas e grau de dificuldade a tabela II nos mostra que dos 190 alunos pesquisados $185(97,36 \%)$ treinaram a técnica de curativo no LHE e somente 46 $(24,21 \%)$ o procedimento de oxigenoterapia. Segundo Pereira et al. ${ }^{9}$ os alunos se interessam pelo aprendizado da técnica do curativo por considerarem ser um campo na qual a enfermagem está diretamente ligada e os resultados desse cuidado pode proporcionar a cura, evitar sofrimentos e complicações. Nas bibliografias consultadas não encontramos dados sobre o pouco treinamento da técnica de oxigenoterapia em LHE, em algumas leituras observamos que os alunos conhecem o uso da oxigenoterapia, porém com pouco conhecimento sobre questões importantes como sinais de hipóxia, o que nos leva a rever a importância do assunto e estimulá-los a um maior treinamento antes da prática clínica. Dos participantes do estudo 123 (64,73\%) afirmaram ter realizado o procedimento de cateterismo vesical masculino e 98 (51,57\%) expressaram ter sentido maior dificuldade na execução dessa técnica. Dificuldade na técnica cataterismo vesical também foi descrito no trabalho de Biazin et al. ${ }^{2}$ onde os alunos atribuíram ao fato de ser uma técnica difícil e em outro estudo realizado por Ohnishi et al. ${ }^{1}$ a técnica de cateterismo vesical também apareceu em menor frequência o que chamou atenção dos docentes por ser uma técnica que exige maior habilidade manual e requerer assepsia 
rigorosa, consequentemente deveria ter um maior treinamento durante as aulas no LHE. Desinfecção do leito e lavagem das mãos não foi citada como dificuldade na realização, provavelmente por serem consideradas técnicas de menor complexidade nessa fase inicial do curso.

A Tabela 2 apresenta as porcentagens das técnicas desenvolvidas no LHE e das técnicas que os alunos mais sentiram dificuldades.

Tabela 2. Eixo habilidades desenvolvidas e grau de dificuldade.

\begin{tabular}{|c|c|c|c|c|c|}
\hline \multirow{2}{*}{$\begin{array}{l}\text { Técnicas desenvolvidas no } \\
\text { laboratório de enfermagem }\end{array}$} & & & \multirow{2}{*}{$\begin{array}{l}\text { Técnicas que os alunos } \\
\text { sentiram mais dificuldades }\end{array}$} & & \\
\hline & $f$ & $\%$ & & $f$ & $\%$ \\
\hline Curativo & 185 & 97,36 & $\begin{array}{l}\text { Cateterismo vesical } \\
\text { masculino } \\
\text { Cateterismo vesical }\end{array}$ & 98 & 51,57 \\
\hline Lavagem das mãos & 183 & 96,31 & feminino & 93 & 48,94 \\
\hline Arrumação de cama & 177 & 93,15 & Exame físico & 66 & 34,73 \\
\hline Verificação de sinais vitais & 176 & 92,63 & Punção venosa & 52 & 27,36 \\
\hline Desinfecção do leito & 175 & 92,10 & Curativo & 46 & 24,21 \\
\hline Banho no leito & 174 & 91,57 & Sondagem nasogástrica & 41 & 21,57 \\
\hline Exame físico & 168 & 88,42 & Sondagem enteral & 35 & 18,42 \\
\hline Colocação de luvas & 165 & 86,84 & Banho no leito & 32 & 16,84 \\
\hline Punção venosa & 145 & 76,31 & Aspiração de vias áereas & 26 & 13,68 \\
\hline Injeção intramuscular & 139 & 73,15 & morte & 21 & 11,05 \\
\hline Preparo do corpo pós-morte & 125 & 65,78 & Injeção intramuscular & 13 & 6,84 \\
\hline Cateterismo vesical masculino & 123 & 64,73 & Injeção subcutânea & 8 & 4,21 \\
\hline Injeção subcutânea & 121 & 63,68 & Colocação de luvas & 6 & 3,15 \\
\hline Sondagem nasogástrica & 116 & 61,05 & Oxigenoterapia & 5 & 2,63 \\
\hline Sondagem enteral & 107 & 56,31 & Verificação de sinais vitais & 5 & 2,63 \\
\hline Cateterismo vesical feminino & 104 & 54,73 & Arrumação de cama & 3 & 1,57 \\
\hline Mensuração de peso e altura & 96 & 50,52 & $\begin{array}{l}\text { Mensuração de peso e } \\
\text { altura }\end{array}$ & 2 & 1,05 \\
\hline Aspiração de vias aéreas & 62 & 32,63 & Desinfecção do leito & 0 & 0 \\
\hline Oxigenoterapia & 46 & 24,21 & Lavagem das mãos & 0 & 0 \\
\hline
\end{tabular}

\section{DISCUSSÃO}

Analisamos no eixo importância do docente durante as práticas o item sobre a demonstração da técnica pelo professor no
LHE antes da prática e 182 alunos (95,78\%) responderam que é válido para o aprendizado e 143 alunos (75,26\%) tiveram os professores auxiliando durante o 
treinamento (Tabela 3). Ohnishi et al. ${ }^{1}$ pesquisando o uso do laboratório no ensino de técnicas fundamentais de enfermagem mostram que $98 \%$ dos alunos também referiram ser válida a metodologia em que o professor demonstra a técnica no laboratório antes da prática clínica e a necessidade de treinarem individualmente, reforçando que habilidade e segurança não se adquire apenas observando. Em estudo de Biazin et al. $^{2}$ avaliando o uso do Laboratório de Enfermagem a maioria dos alunos pesquisados afirmaram que os professores auxiliaram durante $o$ treinamento das técnicas o que corrobora com os resultados do nosso estudo. Em outro estudo de Gomes e Germano $^{5}$ que avaliou a visão dos estudantes no processo ensino/aprendizagem no laboratório de enfermagem destacam o valor do educador em disciplinas que pela primeira vez iniciam conteúdos teórico-práticos, e que os alunos entrevistados expressaram que o professor no laboratório de enfermagem consegue passar melhor o conteúdo, que existe uma melhor interação e que a aula torna-se mais dinâmica, relataram ainda que a confiança e o respeito entre docente e discente é fundamental para o aprendizado uma vez que o aluno não tem medo de expressar suas dúvidas.

A Tabela 3 apresenta as porcentagens da demonstração do procedimento pelo professor no LHE antes da prática e as porcentagens do auxilio dos professores durante o treinamento das técnicas.

Tabela 3. Eixo importância do docente durante as práticas

\begin{tabular}{|c|c|c|c|c|c|}
\hline \multirow{2}{*}{$\begin{array}{l}\text { A demonstração da técnica pelo } \\
\text { professor no laboratório de } \\
\text { enfermagem antes da prática }\end{array}$} & & & \multirow{2}{*}{$\begin{array}{c}\text { Os professores auxiliaram } \\
\text { durante o treinamento das } \\
\text { técnicas }\end{array}$} & \multirow[b]{2}{*}{$f$} & \multirow[b]{2}{*}{$\%$} \\
\hline & $f$ & $\%$ & & & \\
\hline \multicolumn{6}{|l|}{ Valido para o aprendizado } \\
\hline & 182 & $95,78 \%$ & Sim & 143 & $75,26 \%$ \\
\hline $\begin{array}{l}\text { Não é valido para o } \\
\text { aprendizado }\end{array}$ & 5 & $2,63 \%$ & Às vezes & 38 & $20 \%$ \\
\hline Indiferente para o aprendizado & 3 & $1,57 \%$ & Não & 9 & $4,73 \%$ \\
\hline Total & 190 & $100 \%$ & Total & 190 & $100 \%$ \\
\hline
\end{tabular}

Podemos verificar na Tabela IV que 185 alunos $(97,36 \%)$ responderam que a utilização do LHE pela disciplina de Semiologia e Semiotécnica foi importante para seu aprendizado dados semelhantes encontramos no estudo de Ohnishi et al. ${ }^{1}$ que descreveu que $100 \%$ dos alunos participantes da pesquisa afirmaram que 0 
ensino desenvolvido utilizando o laboratório de enfermagem é importante para o aprendizado e em outro estudo de Gomes e Germano $^{5}$ destacam que os alunos foram unânimes em afirmar a importância do laboratório no ensino teórico-prático diminuindo a insegurança dos primeiros cuidados ao cliente.

Foi analisado no eixo percepção do aluno quanto a real utilidade deste tipo de atividade que somente 83 alunos $(43,68 \%)$ utilizaram o LHE para aulas de estudo o que vem de encontro com as respostas de 137 $(72,10 \%)$ participantes da pesquisa que encontraram como fator desestimulante para utilizar o LHE falta de tempo dele para agendar aula de estudo e 93 alunos (48,94\%) responderam que $\mathrm{O}$ LHE ofereceu disponibilidade de horário para estudo. Os horários do LHE pesquisado são organizados de acordo com os cronogramas das disciplinas entregues no início do semestre. Como ele só permanece aberto por dois períodos em cada dia, alternando entre manhã, tarde e noite, são poucos os horários remanescentes para o agendamento de aulas de estudo o que dificulta ainda mais a adesão às práticas, especialmente para aqueles que trabalham. Felix et al. ${ }^{3}$ deixam destacado que $64,7 \%$ dos alunos pesquisados utilizaram o laboratório para reforço de aprendizagem e Ribeiro et al. ${ }^{4}$ pesquisaram a opinião dos alunos de graduação sobre o laboratório de enfermagem na disciplina de centro cirúrgico e $76,5 \%$ retornaram ao laboratório para treinarem as atividades que estavam sendo desenvolvidas no campo de prática.

Os dados mostram que 176 participantes $(92,63 \%)$ concordam que todas as disciplinas teórico-prática do curso devem utilizar o LHE e a necessidade de adquirir maior segurança no procedimento foi um dos fatores que mais estimulou 160 alunos (84,21\%) a utilizarem o LHE durante o semestre em que cursaram a disciplina de Semiologia e Semiotécnica. Em um estudo de Santos e Leite ${ }^{10}$ mostram que a utilização de laboratório de enfermagem e/ou de simulação é cada vez mais utilizada para a formação profissional por ser um método de treinamento seguro, onde o aluno pode treinar e errar, o que não é possível em uma situação real. Gaglizzi e Friedlander ${ }^{7}$ reforçam a grande importância do treinamento prévio no laboratório de enfermagem para o aluno desenvolver maior segurança e competência, diminuindo a ansiedade e medo, reduzindo os riscos ao cliente quando assistido pelo aluno no campo de prática.

A Tabela 4 apresenta as porcentagens da opinião sobre a utilização do LHE pela disciplina de Semiologia e Semiotécnica, bem como a utilização para estudos, disponibilidade de horário, utilização pelas 
Tabela 4. Eixo percepção do aluno quanto a real utilidade deste tipo de atividade

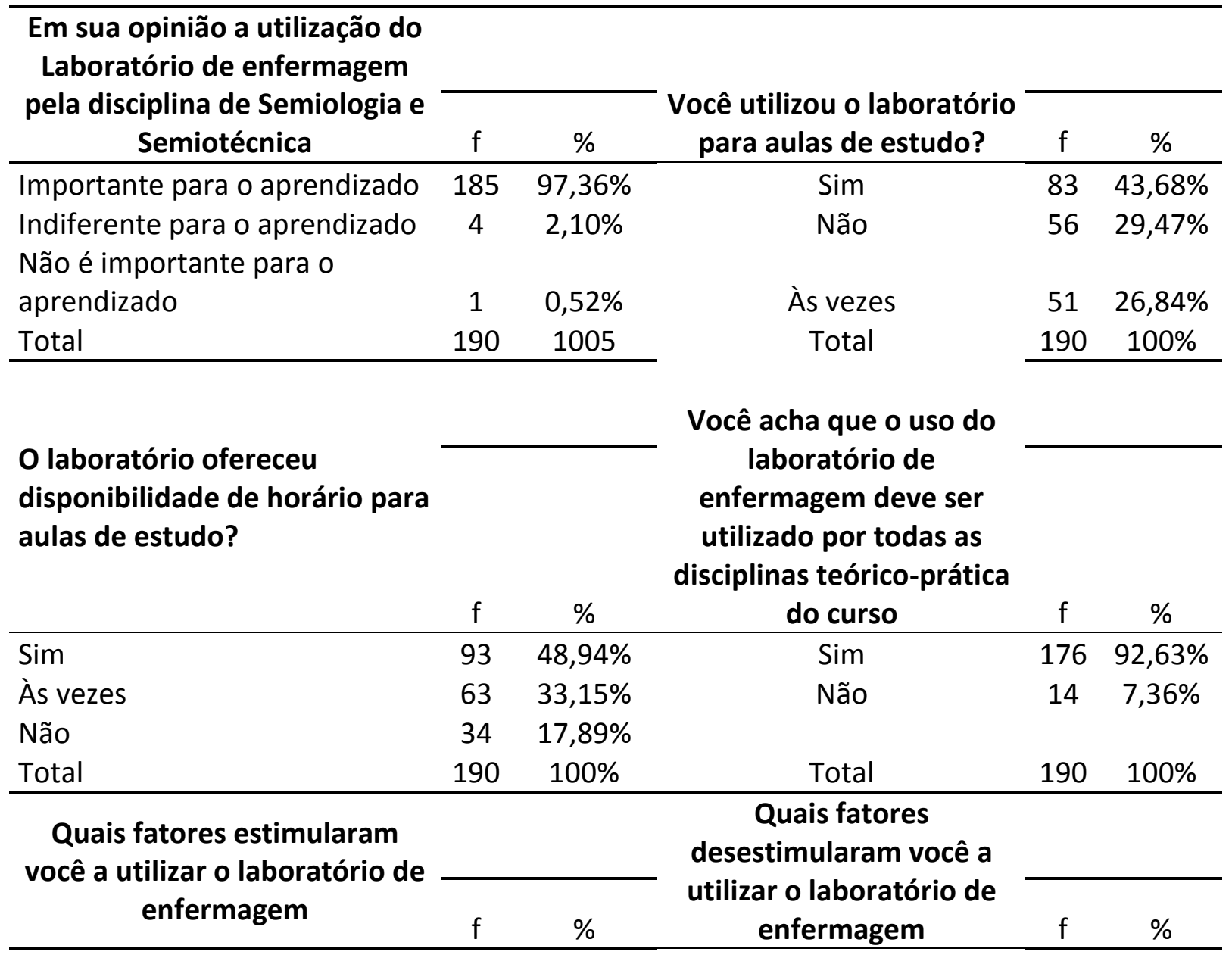

Necessidade de adquirir maior segurança no procedimento

Adquirir conhecimento

Perder o medo

Equipamentos e materiais

suficientes

Curiosidade

Relação aluno professor
$16084,21 \%$

$13973,15 \%$

$94 \quad 49,47 \%$

44

$23,15 \%$

$38 \quad 20 \%$

$38 \quad 20 \%$
Falta de tempo para

agendar aula de estudo

Equipamentos e materiais

insuficientes

$13772,10 \%$

Medo/insegurança

$32 \quad 16,84 \%$

$23 \quad 12,10 \%$

$20 \quad 10,52 \%$

Já conhecer as técnicas a

serem treinadas

$9 \quad 4,73 \%$

Relação aluno-professor

$9 \quad 4,73 \%$ 


\section{CONCLUSÃO}

Para a população avaliada e nas condições propostas neste estudo pode-se concluir que na percepção dos discentes o LHE contribui para sua form/ação acadêmica, especialmente se a participação do docente for efetiva e sugerem ainda que todas as disciplinas teórico-práticas do curso utilizem O LHE.

Quanto aos fatores relacionados à adesão às práticas no LHE a necessidade de adquirir maior segurança no procedimento e aquisição maior conhecimento foram os fatores mais importantes na percepção dos pesquisados e a falta de tempo para agendar aula de estudo foi o fator mais citado como dificuldade na utilização do LHE.

A disponibilização de monitores para auxiliar os alunos durante suas aulas de estudo pode ser um fator que contribua para sua efetiva participação nas aulas, o que foi citado em vários estudos à importância dos mesmos.

Esperamos que os resultados apresentados possam servir de referência para novas pesquisas, evidenciando a importância do laboratório de habilidades de enfermagem como recurso de ensino na formação acadêmica.

\section{Conflito de Interesse}

Os autores declaram não haver qualquer potencial conflito de interesse que possa interferir na imparcialidade deste trabalho cientifico.

\section{REFERÊNCIAS}

1. Ohnishi M, Guariente MHDM, Utyama IKA, Bazoni E. O uso do laboratório no ensino de técnicas fundamentais de enfermagem. Semina: Ciên Biol Saúde. 1995;16(2):272-6. DOI: $\quad$ http://dx.doi.org/10.5433/1679$\underline{0367.1995 v 16 n 2 p 276}$

2. Biazin DT, Guerra AG, Oliveira MC, Santos GM. Uso do laboratório de enfermagem. Terra e Cultura, Ano XVIII. 2002;35:143-162.

3. Felix CCP, Faro ACM, Dias CRF. Percepção de estudantes de enfermagem sobre 0 laboratório de enfermagem como estratégia de ensino. Rev EsC Enferm USP. 2011;45(1):243-9. DOI: http://dx.doi.org/10.1590/S008062342011000100034.

4. Ribeiro RCN, Coutinho RMC, Costa ALS, Amarante ST. Laboratório de enfermagem em centro cirúrgico: opinião de alunos de graduação quanto à sua utilização. Acta Paul Enferm. 1998;11(1):7-13.

5. Gomes CO, Germano RM. Processo ensino/aprendizagem no laboratório de enfermagem: visão de estudantes. Rev Gaúcha Enferm. 2007;28(3):401-8.

6. Gomes, CO. Entre orquídeas e girassóis: o laboratório de enfermagem na visão de estudantes [dissertação]. Natal: Centro de Ciências da Saúde da Universidade Federal do Rio Grande do Norte, Natal; 2004.

7. Gagliazzi MT, Friedlander MR. Ansiedade apresentada pelo estudante na aprendizagem de procedimentos no 
laboratório de enfermagem. Acta Paul Enferm. 2003;16(4):31-7.

8. Teixeira INAO, Felix JVC. Simulação como estratégia de ensino em enfermagem: revisão de literatura. Interface (Botucatu) 2011;15(39):1173-1184.

DOI:

http://dx.doi.org/10.1590/S1414-

$\underline{32832011005000032}$

9. Pereira $A L$, Silva RMP, Silva $M M$, Rezende WL, Pereira AL, Lima EC, et al.Tratamento de feridas: uma contribuição para o ensinoaprendizado. Rev Eletrônica Curso Pedagogia do Campus Jataí-UFG. 2010;2(9).

10. Santos MC, Leite MCL. A avaliação das aprendizagens na prática da simulação em enfermagem como feedback de ensino. Rev Gaúcha Enferm. 2010;31(3):552-6. DOI: http://dx.doi.org/10.1590/S1983-

14472010000300020

Recebido para publicação em 09/04/2014

Revisado em 21/01/2015

Aceito em 16/04/2015 